IFIC/04-25

\title{
Enhanced lepton flavour violation in the supersymmetric inverse seesaw model
}

\author{
F. Deppisch ${ }^{1,2, * *}$ and J. W. F. Valle ${ }^{1,+}$ \\ ${ }^{1}$ AHEP Group, Instituto de Fúsica Corpuscular - C.S.I.C./Universitat de València \\ Edificio Institutos de Paterna, Apt 22085, E-46071 Valencia, Spain \\ ${ }^{2}$ Institut für Theoretische Physik, Universität \\ Würzburg, Am Hubland, D-97074 Würzburg, Germany
}

\begin{abstract}
We discuss a supersymmetric inverse seesaw model in which lepton flavour violating decays can be enhanced either by flavour violating slepton contributions or by the non-unitarity of the charged current mixing matrix. As an example we calculate $\operatorname{Br}(\mu \rightarrow e \gamma)$ taking into account both heavy lepton exchange as well as supersymmetric diagrams in a minimal supergravity framework. We find that the for the same parameters the rate can be enhanced with respect to seesaw model expectations, with or without supersymmetry.

PACS numbers: 11.30.-j, 11.30.Hv, 26.65.+t, 13.15.+g, 14.60.Pq, 95.55.Vj
\end{abstract}

Keywords: supersymmetry; neutrino mass and mixing

${ }^{*}$ Electronic address: deppisch@physik.uni-wuerzburg.de

${ }^{\dagger}$ Electronic address: valle@ific.uv.es 


\section{INTRODUCTION}

A heroic effort dating back to over four decades has finally led to the discovery of neutrino oscillations, through a combination of solar, atmospheric, reactor and accelerator neutrino experiments [1, 2, 3, 4]. This has now firmly established the incompleteness of the Standard Model of electroweak interactions, suggesting that lepton flavour violation (LFV) may also take place in the charged lepton sector.

It is well known that within the framework of the standard non-supersymmetric seesaw models of neutrino masses [5, 6, 6, 8] rare lepton flavour violating decays like $\mu \rightarrow e \gamma$ are indeed extremely rare, far beyond the sensitivity of any foreseeable experiment. In these models the effective light neutrino mass $m_{\nu}$ is inversely proportional to the scale of the lepton number violating $\mathrm{SU}(2) \otimes \mathrm{U}(1)$ singlet Majorana mass $M_{R}, m_{\nu} \propto M_{R}^{-1}$, while typical LFV rates vanish with higher powers of $M_{R}$.

There are two popular mechanisms where lepton flavour violation can be greatly enhanced. First, by supersymmetrizing the model, in which case flavour violation in the neutrino Yukawa couplings is automatically transmitted to charged leptons through charged slepton and sneutrino loops [9, 10, 11], giving a sizeable enhancement to the LFV decay rates.

The other possibility is to consider variants [12] of the seesaw scheme characterized by a small effective lepton number violating Majorana mass term $\mu$ so that $m_{\nu} \propto \mu$. Its smallness may be technically natural, since as $m_{\nu} \rightarrow 0$ a larger symmetry is achieved [13], namely lepton number is restored and neutrinos become massless. Due to the fact that in this case $m_{\nu} \rightarrow 0$ as $\mu \rightarrow 0$ this scheme may be called "inverse seesaw". The resulting LFV rates do not depend at all on the magnitude of the lepton number violating scale $\mu$, which can lie below the weak scale. Moreover, LFV processes may take place even in the limit where lepton number is strictly conserved [14]. Similarly, CP violation can arise in the limit where the light neutrinos are strictly massless [15, 16]. The model thus serves to elucidate that, from a basic point of view, neutrino masses do not play a fundamental role in generating flavour violating processes, which in this case are mediated by the exchange 
of $\mathrm{SU}(2) \otimes \mathrm{U}(1)$ singlet leptons, due to the structure of the electroweak charged and neutral currents [7]. However, in contrast to the standard seesaw, in inverse seesaw schemes the $\mathrm{SU}(2) \otimes \mathrm{U}(1)$ singlet leptons need not be super-heavy, leading to highly enhanced LFV rates irrespective of the massiveness of neutrinos and irrespective of the existence of supersymmetric states [14, 15, 16, 17, 18].

This paper is organized as follows. In Sec. II we introduce the main features of the model, while in Sec. III we give the renormalization group evolution both of the neutrino sector as well as the slepton sector. In Sec. IV] we discuss the relative importance of the two types of contribution to $l_{i}^{-} \rightarrow l_{j}^{-} \gamma$ decays and in Sec. $\nabla$ we give our numerical results and summarize the findings in Sec. VI

\section{INVERSE SEESAW MECHANISM}

The particle content of left-handed leptons in the model extends minimally that of the Standard Model, by the sequential addition of a pair of two-component $\mathrm{SU}(2) \otimes \mathrm{U}(1)$ singlet fermions, as follows

$$
\left(\begin{array}{c}
\nu_{i} \\
e_{i}
\end{array}\right), e_{i}^{c}, \nu_{i}^{c}, S_{i},
$$

with $i$ a generation index running over 1,2,3. In addition to the right-handed neutrinos characteristic of the standard seesaw model, the inverse seesaw scheme contains an equal number of gauge singlet neutrinos $S_{i}$. In the original formulation of the model, these were superstring inspired $E(6)$ singlets, in contrast to the right-handed neutrinos, members of the spinorial representation. A similar construction at the $S U(2)_{L} \otimes S U(2)_{R} \otimes U(1)$ level was considered in Ref. [19].

The model is characterized by the following symmetric $9 \times 9$ mass matrix $\mathcal{M}$ in the $\nu, \nu^{c}, S$ basis,

$$
\mathcal{M}=\left(\begin{array}{ccc}
0 & m_{D}^{T} & 0 \\
m_{D} & 0 & M^{T} \\
0 & M & \mu
\end{array}\right)
$$

where $m_{D}$ and $M$ are arbitrary $3 \times 3$ complex matrices in flavour space, whereas $\mu$ is complex 
symmetric. The matrix $\mathcal{M}$ can be diagonalized by a unitary mixing matrix $U_{\nu}$,

$$
U_{\nu}^{T} \mathcal{M} U_{\nu}=\operatorname{diag}\left(m_{i}, M_{4}, \ldots, M_{9}\right)
$$

yielding 9 mass eigenstates $n_{a}$, three of them corresponding to the observed light neutrinos with masses $m_{i}$, plus the three pairs of two-component leptons $\left(\nu_{i}^{c}, S_{i}\right)$ combining to form three heavy quasi-Dirac leptons [20].

The mass eigenstates are then related to the light neutrino flavour states $\nu_{i}$ via the unitary matrix $U_{\nu}$

$$
\nu_{i}=\sum_{a=1}^{9}\left(U_{\nu}\right)_{i a} n_{a} .
$$

which has been studied in earlier papers [14, 15, 16]. Assuming $m_{D}, \mu \ll M$ the diagonalization results in an effective Majorana mass matrix for the light neutrinos [21],

$$
m_{\nu}=m_{D}^{T} M^{T^{-1}} \mu M^{-1} m_{D}
$$

which may be estimated as

$$
\left(\frac{m_{\nu}}{0.1 \mathrm{eV}}\right)=\left(\frac{m_{D}}{100 \mathrm{GeV}}\right)^{2}\left(\frac{\mu}{1 \mathrm{keV}}\right)\left(\frac{M}{10^{4} \mathrm{GeV}}\right)^{-2},
$$

which vanish in the limit $\mu \rightarrow 0$ where lepton number conservation is restored. In models where lepton number is spontaneously broken by a vacuum expectation value $\langle\sigma\rangle[21]$ one has $\mu=\lambda\langle\sigma\rangle$. For typical Yukawas $\lambda \sim 10^{-3}$ one sees that $\mu=1 \mathrm{keV}$ corresponds to a low scale of $\mathrm{L}$ violation, $\langle\sigma\rangle \sim 1 \mathrm{MeV}$. Although such a low scale is not protected by supersymmetry, this is not needed, as it is already protected by gauge symmetry. Indeed, being a gauge singlet, the smallness of $\langle\sigma\rangle$ is not destabilized by gauge loops and is technically natural ${ }^{1}$.

In such an "inverse seesaw" scheme the three pairs of singlet neutrinos have masses of the order of $M$ and their admixture in the light neutrinos is suppressed as $\frac{m_{D}}{M}$.

In contrast, in the standard seesaw mechanism where the gauge singlet neutrinos $S_{i}$ are absent one would have

$$
\left(\begin{array}{cc}
0 & m_{D}^{T} \\
m_{D} & M_{R}
\end{array}\right), \quad m_{D} \ll M_{R} \Rightarrow m_{\nu}=m_{D}^{T} M_{R}^{-1} m_{D} .
$$

\footnotetext{
${ }^{1}$ For very low values this might lead to interesting signatures in neutrinoless double beta decays 22 .
} 
Note that although $M$ and $M_{R}$ both take the role of a large mass scale suppressing the light neutrino masses, their physical meaning is quite different, the former being a Dirac mass $(\Delta L=0)$ and the latter a Majorana mass $(\Delta L=2)$. In contrast to the mass $M_{R}$ of the right-handed neutrinos of the standard seesaw model, the mass $M$ of our heavy leptons can be much smaller, since the suppression in Eq. (6) is quadratic and since we have the small independent parameter $\mu$ characterizing the violation of lepton number. As a result the value of $M$ may be as low as the weak scale ${ }^{2}$.

Without loss of generality one can assume $\mu$ to be diagonal,

$$
\mu=\operatorname{diag} \mu_{i}
$$

and using the diagonalizing matrix $U$ of the effective light neutrino mass matrix $m_{\nu}$,

$$
U^{T} m_{\nu} U=\operatorname{diag} m_{i}
$$

equation (5) can be written as

$$
\mathbf{1}=\operatorname{diag} \sqrt{m_{i}^{-1}} \cdot U^{T} m_{D}^{T} M^{T^{-1}} \cdot \operatorname{diag} \sqrt{\mu_{i}} \cdot \operatorname{diag} \sqrt{\mu_{i}} \cdot M^{-1} m_{D} U \cdot \operatorname{diag} \sqrt{m_{i}^{-1}} .
$$

In the basis where the charged lepton Yukawa couplings are diagonal the lepton mixing matrix is simply the rectangular matrix formed by the first three rows of $U_{\nu}$ [7].

In analogy to the standard seesaw mechanism [26] it is thus possible to define a complex orthogonal matrix

$$
R=\operatorname{diag} \sqrt{\mu_{i}} \cdot M^{-1} m_{D} U \cdot \operatorname{diag} \sqrt{m_{i}^{-1}}
$$

with 6 real parameters. Using $R$, the neutrino Yukawa coupling matrix $Y_{\nu}=\frac{1}{v \sin \beta} m_{D}$ can be expressed as

$$
Y_{\nu}=\frac{1}{v \sin \beta} M \cdot \operatorname{diag} \sqrt{\mu_{i}^{-1}} \cdot R \cdot \operatorname{diag} \sqrt{m_{i}} \cdot U^{\dagger},
$$

To simplify our discussion we make the assumption that the eigenvalues of both $M$ and $\mu$ are degenerate and that $R$ is real. This allows us to easily compare our results with those obtained previously in Ref. 27, 28] for the case of the standard seesaw mechanism.

\footnotetext{
${ }^{2}$ If light enough, these neutral leptons would be singly-produced in $Z^{0}$ decays 23, 24], a possibility now ruled out by LEP [25].
} 
The combination $Y_{\nu}^{\dagger} L Y_{\nu}$ which is responsible for flavour non-diagonal slepton mass terms in Eqs. (32 34) is given by

$$
\left(Y_{\nu}^{\dagger} L Y_{\nu}\right)_{i j}=\frac{1}{v^{2} \sin ^{2} \beta} \frac{M^{2}}{\mu} \ln \frac{M_{G U T}}{M}\left(U \cdot \operatorname{diag} m_{i} \cdot U^{\dagger}\right)_{i j}
$$

This should be compared with the elements of the light neutrino mass matrix

$$
\left(m_{\nu}\right)_{i j}=\left(U \cdot \operatorname{diag} m_{i} \cdot U^{T}\right)_{i j}
$$

The physical consequence of the simplifications we use is that the pattern of LFV transmitted to the sleptons is closely correlated to that of the light neutrino sector. The only difference being the roles of $\mathrm{CP}$ violating phases in (13) and (14) that may be present in $U$. In the case of $\mathrm{CP}$ conservation which we will consider, the correlation is exact.

In the standard supersymmetric seesaw mechanism the flavour non-diagonal slepton mass terms would be (in an analogous approximation, i.e. $M_{i}=M_{R}$ and real $R$ matrix)

$$
\left(Y_{\nu}^{\dagger} L Y_{\nu}\right)_{i j}=\frac{1}{v^{2} \sin ^{2} \beta} M_{R} \ln \frac{M_{G U T}}{M_{R}}\left(U \cdot \operatorname{diag} m_{i} \cdot U^{\dagger}\right)_{i j}
$$

as compared to Eq. 13.

\section{RENORMALIZATION GROUP EVOLUTION}

\section{A. The neutrino sector}

In what follows it will be sufficient for us to confine ourselves to the simpler case where heavy neutrino masses are degenerate. Below the scale of $M$, the one-loop renormalization group equation (RGE) for the effective neutrino mass matrix in the MSSM is given by [29]

$$
\frac{d}{d t} m_{\nu}=\frac{1}{16 \pi^{2}}\left(\left(-6 g_{2}^{2}-\frac{6}{5} g_{1}^{2}+\operatorname{Tr}\left(6 Y_{U}^{\dagger} Y_{U}\right)\right) m_{\nu}+\left(\left(Y_{l}^{\dagger} Y_{l}\right) m_{\nu}+m_{\nu}\left(Y_{l}^{\dagger} Y_{l}\right)^{T}\right)\right)
$$

with the $\mathrm{U}(1)$ and $\mathrm{SU}(2)$ gauge couplings $g_{1}$ and $g_{2}$, and the Yukawa coupling matrices $Y_{U}$ and $Y_{l}$ for the charge $\frac{2}{3}$-quarks and charged leptons, respectively ${ }^{3}$. This RGE is linear in

\footnotetext{
3 The corresponding evolution equations for $g_{1,2}, Y_{U}$ and $Y_{l}$ can be found in [30].
} 
$m_{\nu}$ and can thus be solved analytically [29] as

$$
m_{\nu}(t)=I(t) m_{\nu}(0) I(t), \quad t=\ln \left(\frac{\mu}{M_{Z}}\right)
$$

Since the evolution is dominated by the gauge and third generation Yukawa couplings one obtains, to a good approximation:

$$
I(t)=I_{g} I_{t} \cdot \operatorname{diag}\left(1,1, I_{\tau}\right)
$$

with

$$
\begin{aligned}
& I_{g}(t)=\exp \left(\frac{1}{16 \pi^{2}} \int_{0}^{t}\left(-3 g_{2}^{2}-\frac{3}{5} g_{1}^{2}\right) d t^{\prime}\right) \\
& I_{t}(t)=\exp \left(\frac{1}{16 \pi^{2}} \int_{0}^{t} 3\left|Y_{t}\right|^{2} d t^{\prime}\right) \\
& I_{\tau}(t)=\exp \left(\frac{1}{16 \pi^{2}} \int_{0}^{t}\left|Y_{\tau}\right|^{2} d t^{\prime}\right)
\end{aligned}
$$

Above the scale $M$, which can be substantially lower than the corresponding heavy lepton scale in standard seesaw schemes, the evolution of the neutrino Yukawa matrix $Y_{\nu}$ is governed by [26]

$$
\frac{d}{d t} Y_{\nu}=\frac{1}{16 \pi^{2}} Y_{\nu}\left(\left(-3 g_{2}^{2}-\frac{3}{5} g_{1}^{2}+\operatorname{Tr}\left(3 Y_{U}^{\dagger} Y_{U}+Y_{\nu}^{\dagger} Y_{\nu}\right)\right) \mathbf{1}+Y_{l}^{\dagger} Y_{l}+3 Y_{\nu}^{\dagger} Y_{\nu}\right)
$$

\section{B. The slepton sector}

Having evolved the neutrino Yukawa couplings to the unification scale $M_{G U T}$, one can now run the slepton mass matrix from $M_{G U T}$ to the electroweak scale assuming the mSUGRA universality conditions on the soft SUSY breaking terms $m_{L}^{2}$ (left-handed slepton doublets), $m_{R}^{2}$ (charged right-handed slepton singlets), $m_{\tilde{N}}^{2}$ (right-handed sneutrino singlets), $A_{e}$ (trilinear couplings analogous to $Y_{e}$ ) and $A_{\nu}$ (trilinear couplings analogous to $Y_{\nu}$ ) at $M_{G U T}$ :

$$
m_{L}^{2}=m_{R}^{2}=m_{\tilde{N}}^{2}=m_{0}^{2} \mathbf{1}, \quad A_{e}=A_{0} Y_{e}, \quad A_{\nu}=A_{0} Y_{\nu}
$$

where $m_{0}$ is the common scalar mass and $A_{0}$ the common trilinear coupling. For definiteness we adopt in our following analysis, the mSUGRA benchmark scenario SPS1a proposed in 
[31], described by

$$
m_{0}=100 \mathrm{GeV}, m_{1 / 2}=250 \mathrm{GeV}, A_{0}=-100 \mathrm{GeV}, \tan \beta=10, \mu>0,
$$

where $\mu$ is here the SUSY Higgs-mixing parameter. In general, the charged slepton (mass) ${ }^{2}$ matrix has the form:

$$
m_{\tilde{l}}^{2}=\left(\begin{array}{cc}
m_{\tilde{l}_{L}}^{2} & \left(m_{\tilde{l}_{L R}}^{2}\right)^{\dagger} \\
m_{\tilde{l}_{L R}}^{2} & m_{\tilde{l}_{R}}^{2}
\end{array}\right),
$$

where $m_{\tilde{l}_{L}}^{2}, m_{\tilde{l}_{R}}^{2}$ and $m_{\tilde{l}_{L R}}^{2}$ are $3 \times 3$ matrices, $m_{\tilde{l}_{L}}^{2}$ and $m_{\tilde{l}_{R}}^{2}$ being hermitian. The matrix elements are given by

$$
\begin{aligned}
\left(m_{\tilde{l}_{L}}^{2}\right)_{a b} & =\left(m_{L}^{2}\right)_{a b}+\delta_{a b}\left(m_{l_{a}}^{2}+m_{Z}^{2} \cos (2 \beta)\left(-\frac{1}{2}+\sin ^{2} \theta_{W}\right)\right) \\
\left(m_{\tilde{l}_{R}}^{2}\right)_{a b} & =\left(m_{R}^{2}\right)_{a b}+\delta_{a b}\left(m_{l_{a}}^{2}-m_{Z}^{2} \cos (2 \beta) \sin ^{2} \theta_{W}\right) \\
\left(m_{\tilde{l}_{L R}}^{2}\right)_{a b} & =\left(A_{\nu}\right)_{a b} v \cos \beta-\delta_{a b} m_{l_{a}} \mu \tan \beta .
\end{aligned}
$$

Applying the mSUGRA conditions (23) at $M_{G U T}$ and performing the evolution to $M_{Z}$ the SUSY breaking terms can be expressed as:

$$
\begin{aligned}
m_{L}^{2} & =m_{0}^{2} \mathbf{1}+\left(\delta m_{L}^{2}\right)_{\mathrm{MSSM}}+\delta m_{L}^{2} \\
m_{R}^{2} & =m_{0}^{2} \mathbf{1}+\left(\delta m_{R}^{2}\right)_{\mathrm{MSSM}}+\delta m_{R}^{2} \\
A_{e} & =A_{0} Y_{e}+\delta A_{\mathrm{MSSM}}+\delta A,
\end{aligned}
$$

with $\left(\delta m_{L, R}^{2}\right)_{\mathrm{MSSM}}$ and $(\delta A)_{\mathrm{MSSM}}$ denoting the usual flavour diagonal MSSM renormalization group corrections [30]. In addition, the presence of right-handed neutrinos radiatively induces flavour off-diagonal terms denoted by $\delta m_{L, R}^{2}$ and $\delta A$. In the leading-log approximation these terms are given as [32]

$$
\begin{aligned}
\delta m_{L}^{2} & =-\frac{1}{8 \pi^{2}}\left(3 m_{0}^{2}+A_{0}^{2}\right)\left(Y_{\nu}^{\dagger} L Y_{\nu}\right) \\
\delta m_{R}^{2} & =0 \\
\delta A & =-\frac{3 A_{0}}{16 \pi^{2}} Y_{e} \cdot\left(Y_{\nu}^{\dagger} L Y_{\nu}\right)
\end{aligned}
$$

with

$$
L=\operatorname{diag}\left(\ln \frac{M_{G U T}}{M_{i}}\right)
$$


Finally, the physical charged slepton masses are then found by diagonalizing (25) using the $6 \times 6$ unitary matrix $U_{\tilde{l}}$ :

$$
U_{\tilde{l}}^{\dagger} m_{\tilde{l}}^{2} U_{\tilde{l}}=\operatorname{diag}\left(m_{\tilde{l}_{1}}^{2}, \ldots, m_{\tilde{l}_{6}}^{2}\right)
$$

Correspondingly, the slepton mass eigenstates are expressed in terms of the gauge eigenstates by

$$
\tilde{l}_{a}=\left(U_{\tilde{l}}^{*}\right)_{i a} \tilde{l}_{L i}+\left(U_{\tilde{l}}^{*}\right)_{(i+3) a} \tilde{l}_{R i}, \quad a=1, \ldots, 6 ; i=e, \mu, \tau .
$$

Similarly to (26) , the $6 \times 6$ (mass) $)^{2}$ matrix of the SUSY partners of the left- and right-handed neutrinos is given by

$$
m_{\tilde{l}}^{2}=\left(\begin{array}{cc}
m_{\tilde{\nu}_{L}}^{2} & \left(m_{\tilde{\nu}_{L R}}^{2}\right)^{\dagger} \\
m_{\tilde{\nu}_{L R}}^{2} & m_{\tilde{\nu}_{R}}^{2}
\end{array}\right)
$$

with

$$
\begin{aligned}
\left(m_{\tilde{\nu}_{L}}^{2}\right)_{a b} & =\left(m_{L}^{2}\right)_{a b}+\frac{1}{2} \delta_{a b} m_{Z}^{2} \cos (2 \beta)+\left(m_{\nu}\right)_{a b}^{2} \\
\left(m_{\tilde{\nu}_{R}}^{2}\right)_{a b} & =\left(m_{\tilde{N}}^{2}\right)_{a b}+M_{a b}^{2} \\
\left(m_{\tilde{\nu}_{L R}}^{2}\right)_{a b} & =v \cos \beta\left(A_{\nu}\right)_{a b}-\mu \cot \beta\left(m_{D}\right)_{a b} .
\end{aligned}
$$

The sneutrino mass matrix is diagonalized by a unitary $6 \times 6$ matrix $U_{\tilde{\nu}}$,

$$
U_{\tilde{\nu}}^{\dagger} m_{\tilde{\nu}}^{2} U_{\tilde{\nu}}=\operatorname{diag}\left(m_{\tilde{\nu}_{1}}^{2}, \ldots, m_{\tilde{\nu}_{6}}^{2}\right)
$$

which, like $U_{\nu}$, can contain sizeable mixings between $\mathrm{SU}(2) \otimes \mathrm{U}(1)$ isodoublet and isosinglet sneutrinos. In contrast to the standard seesaw where the right-handed neutrinos (and thus sneutrinos) are extremely heavy and can be safely neglected in low energy processes, this is not the case in our "inverse seesaw" model. Indeed, the presence of the gauge singlet superfields $S_{i}$ is crucial in this model in causing a big enhancement in the LFV rates. Note however that the scalars present in Eq. (38) can be neglected in calculating the LFV decay rates.

\section{LEPTON FLAVOUR VIOLATING DECAYS: $l_{i}^{-} \rightarrow l_{j}^{-} \gamma$}

The effective Lagrangian for $l_{i}^{-} \rightarrow l_{j}^{-} \gamma$ may be written generically as

$$
\mathcal{L}_{e f f}=\frac{e}{2} \bar{l}_{j} \sigma_{\alpha \beta} F^{\alpha \beta}\left(A_{L}^{i j} P_{L}+A_{R}^{i j} P_{R}\right) l_{i},
$$


where $F^{\alpha \beta}$ is the electromagnetic field strength tensor, $\sigma_{\alpha \beta}=\frac{i}{2}\left[\gamma_{\alpha}, \gamma_{\beta}\right]$ and $P_{R, L}=\frac{1}{2}\left(1 \pm \gamma_{5}\right)$ are the helicity projection operators. The coefficients $A_{L, R}^{i j}$ are determined by the relevant gauge theory Feynman diagrams. The decay rate for $l_{i}^{-} \rightarrow l_{j}^{-} \gamma$ that follows from (43) can be expressed as [33]

$$
\Gamma\left(l_{i}^{-} \rightarrow l_{j}^{-} \gamma\right)=\frac{\alpha}{4} m_{l_{i}}^{3}\left(\left|A_{L}^{i j}\right|^{2}+\left|A_{R}^{i j}\right|^{2}\right) .
$$

\section{A. Heavy lepton contribution}

The contribution to the decay $l_{i}^{-} \rightarrow l_{j}^{-} \gamma$ arising from the admixture of the heavy neutrinos in the left-handed charged current $\mathrm{SU}(2) \otimes \mathrm{U}(1)$ weak interaction 7] exists both for the seesaw scheme as well as for the inverse seesaw model. One finds that the branching ratio is given as 34 ]

$$
\Gamma\left(l_{i}^{-} \rightarrow l_{j}^{-} \gamma\right)=\frac{\alpha_{W}^{3} s_{W}^{2}}{256 \pi^{2}}\left(\frac{m_{l_{i}}}{M_{W}}\right)^{4} \frac{m_{l_{i}}}{\Gamma_{i}}\left|G_{i j}\right|^{2}
$$

where

$$
G_{i j}=\sum_{k}\left(U_{\nu}\right)_{i k}^{*}\left(U_{\nu}\right)_{j k} G_{\gamma}\left(\frac{M_{N_{k}}^{2}}{M_{W}^{2}}\right), \quad G_{\gamma}(x)=-\frac{2 x^{3}+5 x^{2}-x}{4(1-x)^{2}}-\frac{3 x^{3}}{2(1-x)^{4}} \ln x,
$$

$\Gamma_{i}$ is the total decay rate of lepton $i$ and $U_{\nu}$ is the matrix describing the diagonalization of the neutrino mass matrix in the seesaw scheme under consideration. As noted in [14] within the framework of the inverse $\mathrm{SU}(2) \otimes \mathrm{U}(1)$ seesaw mechanism the decay $l_{i}^{-} \rightarrow l_{j}^{-} \gamma$ occurs in the limit of lepton number conservation where the light neutrinos become massless. The rate is correspondingly enhanced with respect to that of the simplest seesaw scheme, as it is not suppressed by the smallness of neutrino masses.

\section{B. Supersymmetric contribution}

The coefficients $A_{L, R}^{i j}$ of the effective Lagrangian for $l_{i}^{-} \rightarrow l_{j}^{-} \gamma$ in Eq. (43) in the MSSM have been given in Ref. [35]. They are determined by the photon penguin diagrams shown in Fig. 1 with charginos/sneutrinos or neutralinos/charged sleptons circulating in the loop. The superscript $c(n)$ refers to the chargino (neutralino) diagram of Fig. 1, while the flavour 

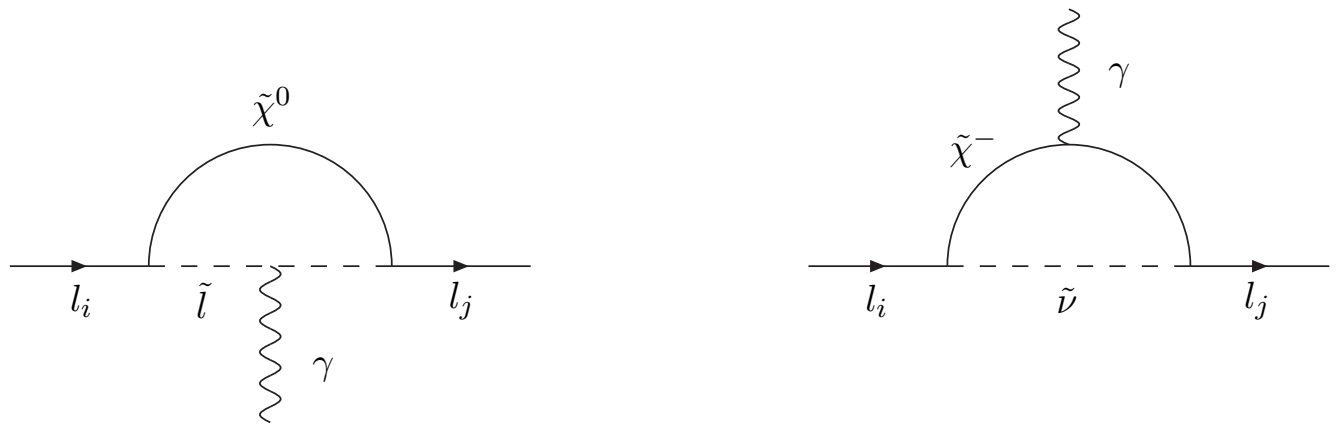

Figure 1: Supersymmetric diagrams for $l_{i}^{-} \rightarrow l_{j}^{-} \gamma$

indices are omitted. Because $m_{l_{i}} \gg m_{l_{j}}$ and $m_{\tilde{l}_{R}}^{2}$ is diagonal (see (2730133)), one has $A_{R} \gg A_{L}[26$, 32]. The dominant amplitudes in (44) are approximately given by

$$
\begin{aligned}
A_{R}^{c} \approx & \frac{1}{32 \pi^{2}} \frac{g_{2}^{2} m_{l_{i}}}{\sqrt{2} m_{W} \cos \beta} \sum_{a=1}^{2} \sum_{k=1}^{6} \frac{m_{\tilde{\chi}_{a}^{-}}}{m_{\tilde{\nu}_{k}}^{2}}\left(O_{R}\right)_{a 1}\left(O_{L}\right)_{a 2}\left(U_{\tilde{\nu}}^{*}\right)_{j k}\left(U_{\tilde{\nu}}\right)_{i k} \\
& \times \frac{-3+4 r_{a k}^{c}-\left(r_{a k}^{c}\right)^{2}-2 \ln r_{a k}^{c}}{\left(1-r_{a k}^{c}\right)^{3}} \\
A_{R}^{n} \approx & -\frac{1}{32 \pi^{2}} g_{2}^{2} \tan \theta_{W} \sum_{a=1}^{4} \sum_{k=1}^{6} \frac{m_{\tilde{\chi}_{a}^{0}}}{m_{\tilde{l}_{k}}^{2}}\left(O_{N}\right)_{a 1}\left(\left(O_{N}\right)_{a 2}+\left(O_{N}\right)_{a 1} \tan \theta_{W}\right) \\
& \times\left(U_{\tilde{l}}^{*}\right)_{j k}\left(U_{\tilde{l}}\right)_{(i+3) k} \frac{1-\left(r_{a k}^{n}\right)^{2}+2 r_{a k}^{n} \ln r_{a k}^{n}}{\left(1-r_{a k}^{n}\right)^{3}}
\end{aligned}
$$

with

$$
r_{a k}^{c}=\left(\frac{m_{\tilde{\chi}_{a}^{-}}}{m_{\tilde{\nu}_{k}}}\right)^{2}, \quad r_{a k}^{n}=\left(\frac{m_{\tilde{\chi}_{a}^{0}}}{m_{\tilde{l}_{k}}}\right)^{2},
$$

the chargino diagonalization matrices $O_{L}, O_{R}$ and the neutralino diagonalization matrix $O_{N}$. The mass eigenvalues of the charginos and neutralinos are denoted by $m_{\tilde{\chi}_{a}^{-}}$and $m_{\tilde{\chi}_{a}^{0}}$, respectively. It is worth noting that the sum in (47) runs over both left- and right-handed sneutrinos. The right-handed sneutrinos will yield a sizeable contribution for small $M$ whereas the left-handed ones and the charged sleptons become significant for larger $M$. The contribution due to flavour non-diagonal slepton mass terms is roughly given by

$$
B r\left(l_{i} \rightarrow l_{j} \gamma\right) \approx \alpha^{3} \tan ^{2} \beta\left(\frac{m_{l_{i}}}{\tilde{m}}\right)^{4} \frac{m_{l_{i}}}{\Gamma_{i}}\left|\frac{\left(\delta m_{L}^{2}\right)_{i j}}{\tilde{m}^{2}}\right|^{2}
$$

where $\tilde{m}$ is the approximate mass scale of SUSY particles in the loops.

The numerical calculations discussed later are performed with the full expressions for $A_{L}^{c, n}$ and $A_{R}^{c, n}$, which can be found in [33] and [36]. 

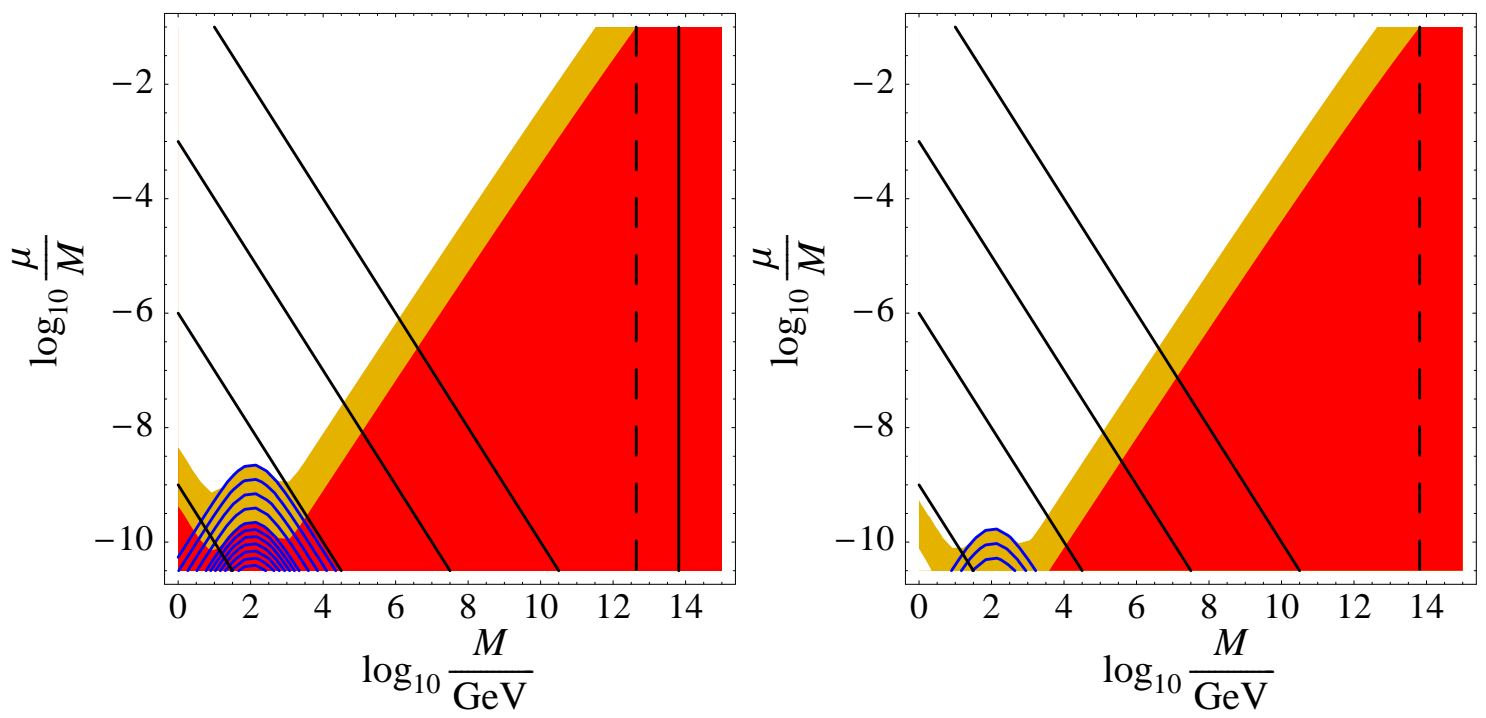

Figure 2: Contours of $\operatorname{Br}(\mu \rightarrow e \gamma)$ in the $\left(M, \frac{\mu}{M}\right)$-plane (logarithmic scales) for hierarchical light neutrinos with $m_{1}=0 \mathrm{eV}$ (left panel) and for degenerate light neutrinos with $m_{1}=0.3 \mathrm{eV}$ (right panel). The dark (light) area is excluded for $\operatorname{Br}(\mu \rightarrow e \gamma)<10^{-11}\left(10^{-13}\right)$. The blue contours in the lower left depict the contribution from neutral heavy leptons only. The diagonal lines show contours of constant $\mu=1,10^{-3}, 10^{-6}, 10^{-9} \mathrm{GeV}$ (top to bottom). The vertical lines are contours of $\operatorname{Br}(\mu \rightarrow e \gamma)$ in the standard SUSY seesaw (see text).

\section{NUMERICAL RESULTS}

Low energy neutrino experiments [1, 2, 3] now provide substantial information on the light neutrino masses and lepton mixing matrix. This information has to be evolved to the unification scale in order to calculate the slepton mass corrections. Our numerical calculation is performed as follows. We fix the light neutrino sector by using the latest global fit [4] for the neutrino oscillation parameters, neglecting possible $\mathrm{CP}$ phases to which current data are insensitive. The Yukawa coupling $Y_{\nu}$ is then calculated via Eq. (12). The result for $\operatorname{Br}(\mu \rightarrow e \gamma)$ in the case of hierarchical neutrino masses, $m_{1}=0 \mathrm{eV}$, is shown in Fig. 2 (left panel) as a contour plot in the $\left(M, \frac{\mu}{M}\right)$-plane, with $\mu<0.1 M$ on the whole plane ${ }^{4}$. The dark (red) area is excluded by the current experimental limit $\operatorname{Br}(\mu \rightarrow e \gamma)<1.2 \cdot 10^{-11}$ while the

\footnotetext{
${ }^{4}$ The diagonal lines depict contours of constant $\mu$ in the inverse seesaw case, for easier reading.
} 
light (brown) band shows the additional sensitivity aimed at the PSI experiment $\operatorname{Br}(\mu \rightarrow$ $e \gamma)<1.5 \cdot 10^{-13}$. For large $M$, the RGE-induced flavour violating slepton contributions are clearly dominant due to the $\left(\frac{M}{\mu / M}\right)^{2} \ln ^{2} \frac{M_{G U T}}{M}$ dependence from Eqs. (13) and (150). It is only below $M \approx 10^{3} \mathrm{GeV}$ where the admixture of the heavy singlet neutrino (and sneutrino) states becomes significant and dominant. In order to guide the eye we have also included the corresponding contours for the standard SUSY seesaw $\mu \rightarrow e \gamma$ decay branching ratio at $10^{-11}$ and $10^{-13}$ (dashed), that would arise from taking $M_{R}=M$, as indicated by the vertical lines on the right side. These are excatly vertical, as the seesaw does not contain the parameter $\mu$. We see that for given $M_{R}=M$ value the rate can be enhanced with respect to seesaw model expectations, with or without supersymmetry.

The curved (blue) contours peaked at slightly above $\mathrm{M}=100 \mathrm{GeV}$ correspond to the nonsupersymmetric isosinglet neutral heavy lepton contribution, considered in [14, 17, 34]. The difference is that now we take into account the neutrino masses indicated by neutrino oscillation data. This actually has no impact, as the violation of flavour in this case arises mainly from the isosinglet neutral heavy lepton contribution, which in this model is essentially unrelated to the light neutrino masses, due to the freedom in choosing the value of $\mu$. On the other hand, in contrast to the above works we explicitly correlate the LFV decay rate to the neutrino oscillation mixing angles. Finally, the rise on the very far left of the plots corresponds the the contribution in the supersymmetric loops arising from $\mathrm{SU}(2) \otimes$ U(1) singlet scalar neutrinos.

The right panel in Fig. 2 shows the analogous plot for quasi-degenerate neutrino masses, $m_{1}=0.3 \mathrm{eV}$, where one can see that $\operatorname{Br}(\mu \rightarrow e \gamma)$ is suppressed by roughly one order of magnitude as compared to the hierarchical neutrino case. This is because the flavour nondiagonal elements of the light neutrino mixing matrix (which are ultimately the source of LFV, both RGE- and non-universality-induced) are suppressed as $\frac{\sqrt{\Delta m_{i j}^{2}}}{m_{1}}$ for large $m_{1}$ 


\section{CONCLUSIONS}

We have discussed a supersymmetric inverse seesaw model in which lepton flavour violating decays can be enhanced either by flavour violating slepton contributions or due to heavy lepton exchange. In contrast to the standard seesaw scheme, the $\mathrm{SU}(2) \otimes \mathrm{U}(1)$ isosinglet heavy leptons present in this model can be relatively light and contribute significantly to LFV processes, irrespective of the magnitude of neutrino masses and irrespective of the supersymmetric contributions. We have considered both types of contributions focusing on their differences with respect to the standard supersymmetric seesaw scheme. As an example we have calculated $\operatorname{Br}(\mu \rightarrow e \gamma)$ taking into account both neutral heavy lepton as well as supersymmetric diagrams in a minimal supergravity framework. Clearly, additional LFV processes such as $\mu \rightarrow 3 e, \mu \rightarrow e$ conversion in nuclei, as well as LFV $\tau$ decays can be considered generalizing to the supersymmetric case the analysis presented in [17, 34].

\section{Acknowledgements}

This work was supported by the Spanish grant BFM2002-00345, by the European Commission Human Potential Programme RTN network HPRN-CT-2000-00148 and by the European Science Foundation network grant N.86. FD was supported by the EU Research Training Site contract HPMT-2000-00124. We thank Martin Hirsch and Heinrich Päs for useful discussions.

[1] Super-Kamiokande Collaboration, Y. Fukuda et al., Phys. Rev. Lett. 81, 1562 (1998), hep-ex/9807003.

[2] SNO Collaboration, Q. R. Ahmad et al., Phys. Rev. Lett. 89, 011301 (2002), nucl-ex/0204008.

[3] KamLAND Collaboration, K. Eguchi et al., Phys. Rev. Lett. 90, 021802 (2003), hep-ex/0212021.

[4] M. Maltoni, T. Schwetz, M. A. Tortola and J. W. F. Valle, hep-ph/0405172, Focus Issue on 
'Neutrino Physics' edited by F.Halzen, M.Lindner and A. Suzuki, to be published in 'New Journal of Physics'. This updates the analysis presented in M. Maltoni et al, Phys. Rev. D68, 113010 (2003), hep-ph/0309130 in view of latest solar and atmospheric flux calculations. Both the review and the paper contain references to analyses by other groups.

[5] M. Gell-Mann, P. Ramond and R. Slansky, (1979), Print-80-0576 (CERN).

[6] T. Yanagida, (KEK lectures, 1979), ed. Sawada and Sugamoto (KEK, 1979).

[7] J. Schechter and J. W. F. Valle, Phys. Rev. D22, 2227 (1980). Phys. Rev. D25, 774 (1982).

[8] R. N. Mohapatra and G. Senjanovic, Phys. Rev. D23, 165 (1981).

[9] L. J. Hall, V. A. Kostelecky and S. Raby, Nucl. Phys. B267, 415 (1986).

[10] F. Borzumati and A. Masiero, Phys. Rev. Lett. 57, 961 (1986).

[11] R. Barbieri and L. J. Hall, Phys. Lett. B338, 212 (1994), hep-ph/9408406.

[12] R. N. Mohapatra and J. W. F. Valle, Phys. Rev. D34, 1642 (1986).

[13] G. 't Hooft, Lecture given at Cargese Summer Inst., Cargese, France, Aug 26 - Sep 8, 1979.

[14] J. Bernabeu et al., Phys. Lett. B187, 303 (1987).

[15] G. C. Branco, M. N. Rebelo and J. W. F. Valle, Phys. Lett. B225, 385 (1989).

[16] N. Rius and J. W. F. Valle, Phys. Lett. B246, 249 (1990).

[17] M. C. Gonzalez-Garcia and J. W. F. Valle, Mod. Phys. Lett. A7, 477 (1992).

[18] J. W. F. Valle, Prog. Part. Nucl. Phys. 26, 91 (1991).

[19] D. Wyler and L. Wolfenstein, Nucl. Phys. B218, 205 (1983).

[20] J. W. F. Valle, Phys. Rev. D27, 1672 (1983). J. W. F. Valle and M. Singer, Phys. Rev. D28, $540(1983)$.

[21] M. C. Gonzalez-Garcia and J. W. F. Valle, Phys. Lett. B216, 360 (1989).

[22] Z. G. Berezhiani, A. Y. Smirnov and J. W. F. Valle, Phys. Lett. B291, 99 (1992), hep-ph/9207209.

[23] M. Dittmar et al., Nucl. Phys. B332, 1 (1990).

[24] M. C. Gonzalez-Garcia, A. Santamaria and J. W. F. Valle, Nucl. Phys. B342, 108 (1990).

[25] DELPHI Collaboration, P. Abreu et al., Z. Phys. C74, 57 (1997).

[26] J. A. Casas and A. Ibarra, Nucl. Phys. B618, 171 (2001), hep-ph/0103065.

[27] F. Deppisch, H. Paes, A. Redelbach, R. Ruckl and Y. Shimizu, Eur. Phys. J. C28, 365 (2003), hep-ph/0206122.

[28] F. Deppisch, H. Pas, A. Redelbach, R. Ruckl and Y. Shimizu, Phys. Rev. D69, 054014 (2004), 
hep-ph/0310053.

[29] J. R. Ellis and S. Lola, Phys. Lett. B458, 310 (1999), hep-ph/9904279.

[30] W. de Boer, Prog. Part. Nucl. Phys. 33, 201 (1994), hep-ph/9402266.

[31] B. C. Allanach et al., Eur. Phys. J. C25, 113 (2002), hep-ph/0202233.

[32] J. Hisano and D. Nomura, Phys. Rev. D59, 116005 (1999), hep-ph/9810479.

[33] J. Hisano, T. Moroi, K. Tobe and M. Yamaguchi, Phys. Rev. D53, 2442 (1996), hep-ph/9510309.

[34] A. Ilakovac and A. Pilaftsis, Nucl. Phys. B437, 491 (1995), hep-ph/9403398.

[35] D. F. Carvalho, J. R. Ellis, M. E. Gomez and S. Lola, Phys. Lett. B515, 323 (2001), hep-ph/0103256.

[36] Y. Okada, K.-i. Okumura and Y. Shimizu, Phys. Rev. D61, 094001 (2000), hep-ph/9906446. 